Vol. 3, No. 2 |Jul - Dec 2019

\title{
A Review of Virtual Touch Interaction Systems
}

\author{
Rizwan Ali Abro", Ahsanullah Abro', Sana Fatima ${ }^{1}$, Sarang Shaikh ${ }^{1}$, Sindhu \\ Abro ${ }^{1}$
}

\begin{abstract}
:
Virtual touch has opened a new dimension in the field of Human Computer Interaction (HCI). Enabled with the motion and gesture detection techniques, it allows manual interaction with virtual environments. Virtual environments are built for performing tasks which are typically achieved using hands in the real world, such as handling objects. An analysis on wearables, graspable and touchable haptic devices used in multiple fields is discussed in this study. This paper reviews the design aspect as well as the usage of the haptic devices along with trendy practices that involved in generating satisfactory touch sensations.
\end{abstract}

Keywords: Virtual Touch, Human Computer Interaction, Review, touch sensations

\section{Introduction}

The sense of touch-Haptics empowers humans to explore and manipulate the tasks with wide variety in real world. The sense of touch must provide the realism to the virtual worlds and robot teleoperation by artificially recreating by stimulated human body (specially the hands). This paper provides the state of the art of design, control and the applications of artificial human sensations that are generated by the haptic devices. There are many areas which can be covered through the haptic technology but the most important to mention in this article is robotic haptics (in this haptic technology the robots enable to get the sense of touch using the force and tactile sensors by using algorithm [1], and invasive sense of touch stimulating haptic (enabling sense of touch-haptic in humans and animals by stimulating peripheral nervous system by electrically [2].

Haptic plays a crucial role and it is quite arduous to expect life without haptics, which is elemental and innate part of life. Sensing and manipulating the real-world objects without haptics imagining the life without water, and can be cause of failure in determining the texture and surface properties [6]. As technology is sphering and growing its impact in all aspects of life as well as business world, the latest technological elements are therefore much needed in every field, for instance, specialized professions can adopt the artificial sense of touch and haptic feedback for remote tasks, astronauts can teleoperate the robots in the space for repairing the space machines, in medical field the surgeons can use the robots in sensitive areas where human hands enable to reach, online customers can sense the stuff of the online products with artificial sense of touch. The human operators are aimed to accord the sense of touch in such teleoperation scenarios, in order to make them feel that they are manipulating the objects and environment like

${ }^{1}$ Department of Computer Science, Sukkur IBA University Sukkur, Sindh, Pakistan Corresponding Author: rizwan.ali@iba-suk.edu.pk 
they interact with the real-world objects with their own hands.

In a few cases, the patients who lost their touch sensation in any disease or accident injury this study has suggested for replacing the sense of touch [11] [12] [13]. Many people lose the sense of touch if an upper-limb amputee is lost because of loss of a hand [14]. In such cases the artificial prosthetic hand used to sense the haptic feedback by interacting with the objects by getting back interaction information from the amputee, as amputee must not rely on the sight itself for manipulating the objects.

The sense of touch is not bounded to a certain area of the human body, contrary to rest four senses (sight, hearing, taste, and smell) [15]. The sense of touch can be found in entire human body particularly tendons, muscles, joints, skin and sensory organs. Usually the sense of touch is split into two modalities: kinesthetic and tactile. The kinesthetic is sensed in the joints, muscles and the tendons, it is usually denominate as torques or forces. While the tactile are purely sensed by the special sensory organs denominate as mechanoreceptors which are present in the skin. It responds to a particular kind of haptic stimulus after sensing from each kind of mechanoreceptor [16] [17] [18] [19] [20].
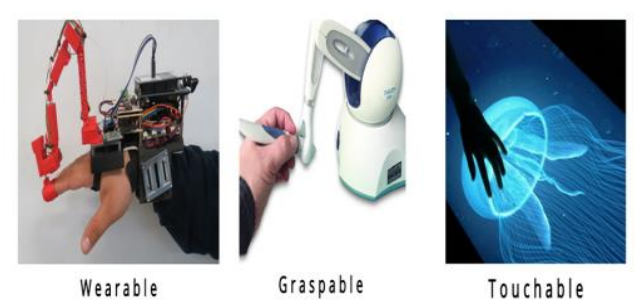

Fig 1: Visual demonstration of Haptic Devices

The mechanoreceptor is restricted and represented to its temporal determination and stick with its susceptible fields [20]. There are two variations in the mechanoreceptors, the fast-adapting mechanoreceptors and the slowadapting mechanoreceptors. The fast-adapting mechanoreceptors is responsible to capture temporary signals while the slow-adapting mechanoreceptors usually capture the static stimuli. For instance, the Meissner corpuscles are example of fast-adapting mechanoreceptors which react with the lower frequency vibrations and be able to sense the skin rate deformation [3].

\section{Literature Review}

The major step to perform the literature review was to collect the recent and the relevant articles from the reliable and identified sources as research questions can be answered. There are three types of haptic devices Graspable, Wearable and touchable [8]. The focus of this review is on all the three categories. furthermore, the presented study is a systematic literature review is on these categories. This study reviews the technology behind the haptic devices in three categories to answer following research questions:

1. What are the most effective and known performance parameters to evaluate the characteristics of haptic devices?

2. What are the most influential devices available in three types of haptic devices (Graspable, Wearable and Touchable)?

The sources which used for the searching the literature were identified by searching the IEEE digital library, ACM library, Springer Link Library and Google Scholar. The searching keywords were "Sense of touch" "Haptic Touch" and "Virtual Touch". The number of conferences and journals were identified are: (IEEE Robotics and

Automation Letter) IEEE RAL, (Benelux Conference on Artificial Intelligence) BNAIC, (ACM on Human-Computer Interaction) ACM HCI, (Virtual, Augmented and Mixed Reality) VAMR, (International Journal of Advanced Robotic Systems) IJARS, (International Conference on Ubiquitous Computing and Ambient Intelligence, Ubiquitous Computing and Ambient Intelligence) UCAmI. 
Table 1: Number of Publications by Source

\begin{tabular}{|l|c|c|c|c|c|c|c|}
\hline Source & $\mathbf{2 0 1 8}$ & $\mathbf{2 0 1 7}$ & $\mathbf{2 0 1 6}$ & $\mathbf{2 0 1 5}$ & $\mathbf{2 0 1 4}$ & $\mathbf{2 0 1 3}$ & Total \\
\hline IEEE RAL & 117 & 188 & 96 & 114 & 132 & 103 & $\mathbf{7 5 0}$ \\
\hline BNAIC & 33 & 29 & 93 & 55 & 81 & 45 & $\mathbf{3 3 6}$ \\
\hline ACM HCI & 179 & 155 & 99 & 125 & 88 & 110 & $\mathbf{7 5 6}$ \\
\hline VAMR & 65 & 45 & 70 & 54 & 82 & 89 & $\mathbf{4 0 5}$ \\
\hline IJARS & 66 & 96 & 145 & 66 & 49 & 111 & $\mathbf{5 3 3}$ \\
\hline UCAmI & 37 & 52 & 61 & 44 & 41 & 54 & $\mathbf{2 8 9}$ \\
\hline
\end{tabular}

Table 2: Number of relevant publications by source

\begin{tabular}{|l|c|c|c|c|c|c|c|}
\hline Source & $\mathbf{2 0 1 8}$ & $\mathbf{2 0 1 7}$ & $\mathbf{2 0 1 6}$ & $\mathbf{2 0 1 5}$ & $\mathbf{2 0 1 4}$ & $\mathbf{2 0 1 3}$ & Total \\
\hline IEEE RAL & 21 & 16 & 31 & 25 & 9 & 15 & $\mathbf{1 1 7}$ \\
\hline BNAIC & 5 & 4 & 18 & 23 & 14 & 13 & $\mathbf{7 7}$ \\
\hline ACM HCI & 8 & 12 & 16 & 9 & 5 & 6 & $\mathbf{5 6}$ \\
\hline VAMR & 11 & 9 & 15 & 14 & 15 & 8 & $\mathbf{7 2}$ \\
\hline IJARS & 4 & 8 & 5 & 11 & 19 & 3 & $\mathbf{5 0}$ \\
\hline UCAmI & 6 & 15 & 14 & 18 & 20 & 16 & $\mathbf{8 9}$ \\
\hline
\end{tabular}

The selection of the papers is based on the most relevant information available in the research paper which addresses the usage of the haptic devices based on said categories. The sources were extracted based on the keywords selection which matched this papers requirement. There were 70 papers included in this study but later the number was decreased because this study has to address and answer the research questions. The literature review was narrowed based on the publications between the years 2013 to 2018 because of need of the haptic devices in the said period. Table 1 highlighted the publications numbers which have been examined from each source. The sources used in this study has different numbers of publications, such as, (Journal Articles, short papers, Full papers). this study focuses on the conference and journal articles.

The haptic word is derived from the Greek word "haptesthai" which is described as the ability to manipulate or touch the objects [55]. There are two parts of the haptic systems which are human and machine parts. The human part is responsible for sensing and controlling the position of hand whereas machine part helps to simulate the contact with a virtual object by exerting forces from the hand [56].

extensive use of the wearable devices which make use of the haptic rendering techniques that allows users to manipulate variety of objects. For example, a simple haptics aided glove paired with wrist sensors can assist to have the sense of wrist orientation. With the help of virtual reality patients are made to 
perform the motion related tasks during training sessions to reach the goals of increasing complexity [57]. Haptic technology was introduced by military field specially for astronauts and pilots' training simulation. As the advancements in computer technologies took place gaming industry began to use this technology in video games such as oculus rift, which is an example of wearable device [58].

Increasing usage of graspable devices has been witnessed in medical training sessions as well as in surgeries as the use of VR Robotic technology has enhanced [59].

Haptic system in robotic technology is considered to be a channel that can provide force sensations from real environment to the users. This system is referred to the handling and application of touch sensation to interact with machines. such as haptic telepresence robots or other touchable devices are considered in this category [60].

In order to provide sensory feedback, haptic applications are equipped with specialized hardware. Commonly used haptic interface configuration uses the mechanical linkages to connect a person's finger with computer interface. Whenever user moves its finger the translated actions are highlighted on the screen [61].

\section{Results and Discussions}

The paper discussed the various aspects in haptic devices which are highlighted and listed first in the literature. Many haptic devices are available in all three categories. The usage of the haptic devices for various purposes which includes the most commonly defined property such as availability of the devices, which devices has most impact and which device is preferred by the user based on reliability and price. It is obvious that haptic device ought to fascinate the user requirements by considering the reliability and prices factors. Many researchers have addressed this issue that the device functionality is directly proportional to its price [14].
There is a significant increase in the use of technology in almost every field so it is required to switch the number of aesthetically potential applications to haptics. The use of haptics is witnessed in communication, online stores, medical and gaming [35]. Throughout the study it has seen that impact of the haptics in different areas where the human interaction is almost impossible or hard to tackle the situation, the sense of touch is welcoming to play its role in those situations, for instance, robotic distance surgery, prosthetic organs) that touch has ability to replace the visual or auditory senses cues and touch can augment the virtual interactions. One of the major limiting reasons in the field of haptics is lack of availability of hardware and its expressiveness. Limited number of actuators available commercially for designing haptic output exclusively and it bring many researchers to make the haptic devices at their own using off the shelf components [38,42]. Focusing on the perceptual abilities of human sense of touch during the process of design is a key to develop functional as well as effective haptic hardware. The human centered design paradigm leads in more effective stimulation of haptic hardware that mechanoreceptors to visualize the required sensations. The primary focus of this article to extract the research questions from the study and to answer those questions from the driven results and how important is each factor of graspable, wearable and touchable.

In order to identify the evaluation known factors in order to evaluate the performance factors in haptic devices. Many research papers have discussed various methodologies to evaluate them. In early period of haptic touch, the touchable devices were the most dominating technology with passage of time the dominated factor changed to the graspable devices they quite popular in gaming joysticks. In recent times the wearable devices have been introduced and using in haptics [50]. The Virtual Reality is dominated by the wearable devices. based on the study it has been found that the usage of wearable haptic

Sukkur IBA Journal of Computing and Mathematical Science - SJCMS | Vol. 3 No. 2 July - December 2019 @ Sukkur IBA University 
devices is increased from 2015 and onwards and the rest two categories still have impacted the touchable devices are being used widely for sense of touch purposes [19]. The graspable devices have a smaller number of users and impact over the two categories.

\section{Conclusion}

The role of the technology has seen such a dominating aspect in communication, online shopping and entertainment increases and growing number of potential applications of sense of touch. The haptics has gained the promising familiarity in terms of usage in mobile communication, gaming as well as in real world applications, although the limit in commercial haptic devices is seen. The impact of the haptics has increased especially in those areas where physical sense of touch is critical to perform the task (e.g., remote surgeries). where touch can be replaced and suggests in terms of visual or auditory cues (e.g., mobile communication and navigation), and where touch can enhance virtual interactions (e.g., virtual reality and gaming). It has been observed that the wearable devices are used most preferred in above cases and the reason is that it is very easy to wear and its hardware can easily be found in the market.

Although limitations of using haptic technology includes lack of availability of hardware but still, haptic, "sense of touch" technology, is expected to gain prominence in the near future as it facilitates humancomputer interaction and offers better contact and detailed information about a real-world object. The prolific use of this technology in automotive, education, and gaming is likely to create new opportunities for the global technology market. In addition, the demand for innovative consumer electronics products such as smartphones and tablets are also expected to be a key driver of market growth.

\section{REFERENCES}

[1] Chu V, McMahon I, Riano L, McDonald CG, He Q, et al. 2015. Robotic learning of haptic adjectives

through physical interaction. Robot. Auton. Syst. 63:279-92

[2] Flesher SN, Collinger JL, Foldes ST, Weiss JM, Downey JE, et al. 2016. Intracortical microstimulation of human somatosensory cortex. Sci. Transl. Med. 8:361ra141

[3] Johansson RS, Flanagan JR. 2009. Coding and use of tactile signals from the fingertips in object manipulation tasks. Nat. Rev. Neurosci. 10:345-59

[4] Johnson KO, Yoshioka T, Vega-Bermudez F. 2000. Tactile functions of mechanoreceptive afferents innervating the hand. J. Clin. Neurophysiol. 17:539-58

[5] Bolanowski SJ, Gescheider GA, Verrillo RT. 1994. Hairy skin: psychophysical channels and their physiological substrates. Somatosens. Motor Res. 11:279-90

[6] Yohanan, Steve, and Karon E. MacLean. "The role of affective touch in human-robot interaction: Human intent and expectations in touching the haptic creature." International Journal of Social Robotics 4.2 (2012): 163180

[7] Bushra, N., Carruth, D., \& Deb, S. (2018). A Comparative Study of Virtual UI for Risk Assessment and Evaluation. In Advances in Visual Computing (pp. 226-236). Springer International Publishing.

[8] Goedschalk, L., Bosse, T., \& Otte, M. (2018). Get Your Virtual Hands Off Me! Developing Threatening IVAs Using Haptic Feedback. In Communications in Computer and Information Science (pp. 61-75). Springer International Publishing.

[9] Zhao, Y., Ham, J., \& van der Vlist, J. (2018). Persuasive Virtual Touch: The Effect of Artificial Social Touch on Shopping Behavior in Virtual Reality. In Symbiotic Interaction (pp. 98-109). Springer International Publishing

[10] Barreiros, J., Claure, H., Peele, B., Shapira, O., Spjut, J., Luebke, D., ... Shepherd, R. (2019). Fluidic Elastomer Actuators for Haptic Interactions in Virtual Reality. IEEE Robotics and Automation Letters, 4(2), 277284.

[11] Chaudhuri, S., \& Priyadarshini, K. (2017). Cultural Heritage Objects: Bringing Them Alive Through Virtual Touch. In Digital 
Hampi: Preserving Indian Cultural Heritage (pp. 337-354). Springer Singapore.

[12] Culbertson, H., Nunez, C. M., Israr, A., Lau, F., Abnousi, F., \& Okamura, A. M. (2018). A social haptic device to create continuous lateral motion using sequential normal indentation. In 2018 IEEE Haptics Symposium (HAPTICS). IEEE.

[13] Haritaipan, L., Hayashi, M., \& Mougenot, C. (2018). Design of a Massage-Inspired Haptic Device for Interpersonal Connection in LongDistance Communication. Advances in Human-Computer Interaction, 2018, 1-11.

[14] Nakanishi, J., Sumioka, H., \& Ishiguro, H. (2018). Virtual Hug Induces Modulated Impression on Hearsay Information. In Proceedings of the 6th International Conference on Human-Agent Interaction HAI '18. ACM Press.

[15] Dombrowski, M., \& Dombrowski, J. (2017). Virtual Reality Games, Therapeutic Play and Digital Healing. In Distributed, Ambient and Pervasive Interactions (pp. 587-596). Springer International Publishing

[16] Lee, Y., \& Kim, G. J. (2017). Vitty: Virtual Touch Typing Interface with Added Finger Buttons. In Lecture Notes in Computer Science (pp. 111-119). Springer International Publishing.

[17] Kim, H., Kim, M., \& Lee, W. (2016). HapThimble. In Proceedings of the 2016 CHI Conference on Human Factors in Computing Systems - CHI '16. ACM Press.

[18] Hoshina, A., Okada, Y., Giannopulu, I., \& Sugaya, M. (2016). Haptic Virtual Approach: Biological Effect on Touching and Viewing. In Lecture Notes in Computer Science (pp. 261-270). Springer International Publishing

[19] Cheok, A. D., \& Pradana, G. A. (2015). Virtual Touch. In Scholarpedia of Touch (pp. 837-849). Atlantis Press.

[20] Yarosh, S., Mejia, K., Unver, B., Wang, X., Yao, Y., Campbell, A., \& Holschuh, B. (2017). SqueezeBands. Proceedings of the ACM on Human-Computer Interaction, 1(CSCW), 1-18.

[21] Takahashi, H., Ban, M., Osawa, H., Nakanishi, J., Sumioka, H., \& Ishiguro, H. (2017). Huggable Communication Medium Maintains Level of Trust during Conversation Game. Frontiers in Psychology, 8

[22] Shiomi, M., Nakata, A., Kanbara, M., \& Hagita, N. (2017). A hug from a robot encourages prosocial behavior. In 2017 26th IEEE International Symposium on Robot and Human Interactive Communication (ROMAN). IEEE.

[23] Mejia, K., \& Yarosh, S. (2017). A Nine-Item Questionnaire for Measuring the Social Disfordance of Mediated Social Touch Technologies. Proceedings of the ACM on Human-Computer Interaction, 1(CSCW), 117.

[24] Marshall, J., \& Tennent, P. (2017). Touchomatic. In Proceedings of the 2017 Conference on Designing Interactive Systems - DIS '17. ACM Press

[25] Sajadieh, S. (2017). Touch Me Here. In Proceedings of the 2017 ACM on Multimedia Conference - MM '17. ACM Press.

[26] Gugenheimer, J., Dobbelstein, D., Winkler, C., Haas, G., \& Rukzio, E. (2016). FaceTouch. In Proceedings of the 29th Annual Symposium on User Interface Software and Technology - UIST '16. ACM Press

[27] Ahmed, I., Harjunen, V., Jacucci, G., Hoggan, E., Ravaja, N., \& Spapé, M. M. (2016). Reach out and touch me: effects of four distinct haptic technologies on affective touch in virtual reality. In Proceedings of the 18th ACM International Conference on Multimodal Interaction - ICMI 2016. ACM Press.

[28] Ahmed, I., Harjunen, V., Jacucci, G., Hoggan, E., Ravaja, N., \& Spapé, M. M. (2016). Reach out and touch me: effects of four distinct haptic technologies on affective touch in virtual reality. In Proceedings of the 18th ACM International Conference on Multimodal Interaction - ICMI 2016. ACM Press.

[29] Rasool, S., Hou, X., Liu, Y., Sourin, A., \& Sourina, O. (2016). Assessing haptic video interaction with neurocognitive tools. In 2016 IEEE International Conference on Systems, Man, and Cybernetics (SMC). IEEE.

[30] Misawa, K., \& Rekimoto, J. (2016). Who am I Touching? In Proceedings of the Fourth International Conference on Human Agent Interaction - HAI '16. ACM Press

[31] Canat, M., Tezcan, M. O., Yurdakul, C., Tiza, E., Sefercik, B. C., Bostan, I., ... Özcan, O. (2016). Sensation. In Proceedings of the 2016 CHI Conference on Human Factors in Computing Systems - CHI '16. ACM Press. 
[32] Maike, V. R. M. L., de Sousa Britto Neto, L., Baranauskas, M. C. C., \& Goldenstein, S. K. (2014). Seeing through the Kinect: A Survey on Heuristics for Building Natural User Interfaces Environments. In Universal Access in Human-Computer Interaction. Design and Development Methods for Universal Access (pp. 407-418). Springer International Publishing.

[33] Huisman, G., Bruijnes, M., Kolkmeier, J., Jung, M., Darriba Frederiks, A., \& Rybarczyk, Y. (2014). Touching Virtual Agents: Embodiment and Mind. In Innovative and Creative Developments in Multimodal Interaction Systems (pp. 114-138). Springer Berlin Heidelberg.

[34] Wang, J., Vasisht, D., \& Katabi, D. (2014). RF-IDraw. ACM SIGCOMM Computer Communication Review, 44(4), 235-246.

[35] Mateu, J., Lasala Bello, M. J., \& Alamán, X. (2014). Virtual Touch Book: A Mixed-Reality Book for Inclusive Education. In Ubiquitous Computing and Ambient Intelligence. Personalisation and User Adapted Services (pp. 124-127). Springer International Publishing.

[36] Mei, K., Feng, S., Li, G., Chou, W., \& Zhang, L. (2013). A virtual touch event method using scene recognition for digital television. IEEE Transactions on Consumer Electronics, 59(1), 214-219

[37] Corbett, B., Yamaguchi, T., Liu, S., Huang, L., Bahn, S., \& Nam, C. S. (2013). Influence of Haptic Feedback on a Pointing Task in a Haptically Enhanced 3D Virtual Environment. In Human-Computer Interaction. Interaction Modalities and Techniques (pp. 561-567). Springer Berlin Heidelberg.

[38] Monnai, Y., Hasegawa, K., Fujiwara, M., Yoshino, K., Inoue, S., \& Shinoda, H. (2014). HaptoMime. In Proceedings of the 27th annual ACM symposium on User interface software and technology - UIST '14. ACM Press.

[39] Angelini, L., Khaled, O. A., Caon, M., Mugellini, E., \& Lalanne, D. (2014). Hugginess. In Proceedings of the 2014 ACM International Symposium on Wearable Computers Adjunct Program - ISWC '14 Adjunct. ACM Press.

[40] Overmars, S., \& Poels, K. (2013). Virtual Touch Sensations in an Online Shopping
Context: An Experimental Approach. In 2013 Humaine Association Conference on Affective Computing and Intelligent Interaction. IEEE.

[41] Huang, Y.-P., Wang, G.-Z., Chang, T.-S., \& Chen, T.-H. (2013). Three-Dimensional Virtual Touch Display System for Multi-User Applications. Journal of Display Technology, 9(11), 921-928.

[42] Philpott, M., \& Summers, I. R. (2012). Evaluating a Multipoint Tactile Renderer for Virtual Textured Surfaces. In Haptics: Perception, Devices, Mobility, and Communication (pp. 121-126). Springer Berlin Heidelberg.

[43] Cuartielles, D., Göransson, A., Olsson, T., \& Stenslie, S. (2012). Mobile Haptic Technology Development through Artistic Exploration. In Haptic and Audio Interaction Design (pp. 31-40). Springer Berlin Heidelberg

[44] Cheok, A. D. (2010). Huggy Pajama: A Remote Interactive Touch and Hugging System. In Art and Technology of Entertainment Computing and Communication (pp. 161-194). Springer London.

[45] Bickmore, T. W., Fernando, R., Ring, L., \& Schulman, D. (2010). Empathic Touch by Relational Agents. IEEE Transactions on Affective Computing, 1(1), 60-71.

[46] Zhao, Y., \& Dai, S. (2009). A Robust and Fast Monocular-Vision-Based Hand Tracking Method for Virtual Touch Screen. In 2009 2nd International Congress on Image and Signal Processing. IEEE.

[47] Ahn, Y.-K., Park, Y.-C., Choi, K.-S., Park, W.-C., Seo, H.-M., \& Jung, K.-M. (2009). TOF Depth Camera Based 3D Gesture Interaction System. In Advances in Information Technology (pp. 11-17). Springer Berlin Heidelberg.

[48] Bickmore, T. W., Fernando, R., Ring, L., \& Schulman, D. (2010). Empathic Touch by Relational Agents. IEEE Transactions on Affective Computing, 1(1), 60-71.

[49] Bailenson, J. N., \& Yee, N. (2007). Virtual interpersonal touch: Haptic interaction and copresence in collaborative virtual environments. Multimedia Tools and Applications, 37(1), 5-14.

[50] Lee, S. P., Cheok, A. D., James, T. K. S., Debra, G. P. L., Jie, C. W., Chuang, W., \& 
Farbiz, F. (2005). A mobile pet wearable computer and mixed reality system for human-poultry interaction through the internet. Personal and Ubiquitous Computing, 10(5), 301-317

[51] Bailenson, J. N., \& Yee, N. (2007). Virtual interpersonal touch: Haptic interaction and copresence in collaborative virtual environments. Multimedia Tools and Applications, 37(1), 5-14.

[52] Konyo, M., Tadokoro, S., Yoshida, A., \& Saiwaki, N. (2005). A tactile synthesis method using multiple frequency vibrations for representing virtual touch. In 2005 IEEE/RSJ International Conference on Intelligent Robots and Systems. IEEE.

[53] Koskela, T., \& Vilpola, I. (2004). Usability of MobiVR Concept: Towards Large Virtual Touch Screen for Mobile Devices. In Mobile Human-Computer Interaction - MobileHCI 2004 (pp. 252-263). Springer Berlin Heidelberg.

[54] Dionisio, J., Henrich, V., Jakob, U., Rettig, A., \& Ziegler, R. (1997). The virtual touch: Haptic interfaces in virtual environments. Computers \& Graphics, 21(4), 459-468.

[55] Sreelakshmi, M., and T. D. Subash. "Haptic technology: A comprehensive review on its applications and future prospects." Materials Today: Proceedings 4.2 (2017): 4182-4187.
[56] El Saddik, Abdulmotaleb. "The potential of haptics technologies." IEEE Instrumentation \& Measurement Magazine 10.1 (2007): 1017.

[57] D'Auria, Daniela, Fabio Persia, and Bruno Siciliano. "A low-cost haptic system for wrist rehabilitation." 2015 IEEE International Conference on Information Reuse and Integration. IEEE, 2015.

[58] Liu, Lu-miao, Wei Li, and Jia-jia Dai. "Haptic technology and its application in education and learning." 2017 10th International Conference on Ubi-media Computing and Workshops (Ubi-Media). IEEE, 2017.

[59] Agboh, Wisdom C., Mustafa Yalcin, and Volkan Patoglu. "A six degrees of freedom haptic interface for laparoscopic training." 2016 IEEE/RSJ International Conference on Intelligent Robots and Systems (IROS). IEEE, 2016.

[60] Muis, Abdul, and Kouhei Ohnishi. "Cooperative mobile manipulator with dual compliance controllers based on estimated torque and visual force." 2006 IEEE International Conference on Mechatronics. IEEE, 2006.

[61] Salisbury, Kenneth, Francois Conti, and Federico Barbagli. "Haptic rendering: introductory concepts." IEEE computer graphics and applications 24.2 (2004): 24-32. 